OPEN ACCESS

Edited by: Ashok Kumar,

University of Florida, USA

Reviewed by:

Karthik Bodhinathan,

Sanford Burnham Medical Research

Institute, USA

Olivier Thibault,

University of Kentucky, USA

*Correspondence: Mario Marchi,

Department of Pharmacy,

Pharmacology and Toxicology

Section, University of Genoa, Viale

Cembrano 4, 16148 Genoa, Italy

marchi@pharmatox.unige.it

Specialty section: This article was submitted to

Neuropharmacology,

a section of the journal

Frontiers in Pharmacology

Received: 03 March 2015

Accepted: 10 April 2015

Published: 29 April 2015

Citation:

Marchi M, Grilli M and Pittaluga AM

(2015) Nicotinic modulation of glutamate receptor function at nerve terminal level: a fine-tuning of synaptic

signals.

Front. Pharmacol. 6:89. doi: 10.3389/fphar.2015.00089

\section{Nicotinic modulation of glutamate receptor function at nerve terminal level: a fine-tuning of synaptic signals}

\author{
Mario Marchi ${ }^{1,2 *}$, Massimo Grilli ${ }^{1}$ and Anna M. Pittaluga ${ }^{1,2}$ \\ ${ }^{1}$ Department of Pharmacy, Pharmacology and Toxicology Section, University of Genoa, Genoa, Italy, ${ }^{2}$ Center of Excellence \\ for Biomedical Research, University of Genoa, Genoa, Italy
}

This review focuses on a specific interaction occurring between the nicotinic cholinergic receptors (nAChRs) and the glutamatergic receptors (GluRs) at the nerve endings level. We have employed synaptosomes in superfusion and supplemented and integrated our findings with data obtained using techniques from molecular biology and immunocytochemistry, and the assessment of receptor trafficking. In particular, we characterize the following: (1) the direct and unequivocal localization of native $\alpha$-amino-3-hydroxy5-methyl-4-isoxazolepropionic acid (AMPA) and N-methyl-D-aspartate (NMDA) glutamatergic receptors on specific nerve terminals, (2) their pharmacological characterization and functional co-localization with nAChRs on the same nerve endings, and (3) the existence of synergistic or antagonistic interactions among them. Indeed, in the rat nucleus accumbens (NAc), the function of some AMPA and NMDA receptors present on the dopaminergic and glutamatergic nerve terminals can be regulated negatively or positively in response to a brief activation of nAChRs. This effect occurs rapidly and involves the trafficking of AMPA and NMDA receptors. The event takes place also at very low concentrations of nicotine and involves the activation of several nAChRs subtypes. This dynamic control by cholinergic nicotinic system of glutamatergic NMDA and AMPA receptors might therefore represent an important neuronal presynaptic adaptation associated with nicotine administration. The understanding of the role of these nicotineinduced functional changes might open new and interesting perspectives both in terms of explaining the mechanisms that underlie some of the effects of nicotine addiction and in the development of new drugs for smoking cessation.

Keywords: nicotinic receptors, AMPA receptors, NMDA receptors, synaptosomes, neurotransmitter release, receptor-receptor interactions, synaptic plasticity

\section{Introduction}

Neuronal nicotinic receptors (nAChRs) are widely distributed in the rats' central nervous system (CNS). They are located on different types of neurons, and are thought to serve different functions (Dajas-Bailador and Wonnacott, 2004). Accordingly, the outcome of the nAChRs stimulation depends on both the location of the cholinergic neurons and the specific type of neuron on which

Abbreviations: AMPA, $\alpha$-amino-3-hydroxy-5-methyl-4-isoxazolepropionic acid; CNS, central nervous system; DA, dopamine; GABA, $\gamma$-aminobutyric acid; GluN2B/GluN2A, Glutamate N2B/N2A subunits; NAc, nucleus accumbens; nAChR, nicotinic acetylcholine receptor; NMDA, $N$-methyl-D-aspartate; NMDARs, NMDA receptors. 
the receptors are expressed. Data from studies performed with genetically modified mice combined with neurotransmitter release and electrophysiological data consistently show that nAChRs exert a predominant role in the modulation of the release of neurotransmitters in the brain (Wonnacott, 1997).

Certainly, one of the most studied roles of nAChRs present on nerve endings is their ability to facilitate the release of several neurotransmitters (Arqueros et al., 1978; Wonnacott, 1997; Sher et al., 2004; Jones and Wonnacott, 2005; Marchi and Grilli, 2010). The effect of nicotine occurs throughout the activation of nAChR subtypes, which have different pharmacological and functional characteristics. One of the most abundant nAChRs present in the CNS, the $\alpha 4 \beta 2 \mathrm{nAChR}$ subtype, favors the entry of sodium and causes the depolarization that in turn activates the voltage operated calcium channels and thus facilitates the entry of calcium (Wonnacott, 1997; Dajas-Bailador and Wonnacott, 2004). Others, such as the $\alpha 7 \mathrm{nAChR}$ subtypes, are much more permeable to calcium (Bertrand et al., 1990) which when present in the cytoplasmmay activate the mobilization of calcium from intracellular stores (Dickinson et al., 2008). Throughout both of these mechanisms, nicotine evokes the release of several neurotransmitters (Turner, 2004; Zappettini et al., 2010). The $\mathrm{EC}_{50}$ value of nicotine falls within a micromolar range (McGehee and Role, 1995; Vibat et al., 1995; Buisson et al., 1996), while at concentrations equal to or less than $0.1 \mu \mathrm{M}$ nicotine is really little or non-effective (Marchi et al., 2002; Zappettini et al., 2011).

However, quite interestingly, nicotine, may also modulate the functional responses of some receptors co-existing with nAChRs on the same nerve ending (Vizi and Lendvai, 1999; Sher et al., 2004; Parodi et al., 2006; Patti et al., 2006; Grilli et al., 2008, 2009a,b; Lin et al., 2010; Marchi and Grilli, 2010). This ability of nicotine in controlling receptor-mediated responses is relatively new and particularly intriguing when considering that sometimes it occurs also at nicotine concentrations insufficient to elicit neurotransmitter release. Briefly, the mechanism of receptor-receptor cross talk escapes that of control of neurotransmitter exocytosis but it finely tunes those mechanisms controlling in-out movements of receptors in synaptosomal plasma membranes. In particular, at the level of dopaminergic terminals, nicotine may exert a negative modulatory effect on the functionality of other receptors, coexisting with $\mathrm{nAChRs}$, and this gives rise to a decrease of dopamine (DA) release if elicited by other neurotransmitter systems. Accordingly, nicotine used at low concentrations does not produce its typical stimulatory effect on DA release but conversely may cause inhibitory effects..

The modifications of receptor function sometimes are the outcome of a direct structural interaction between the two receptor systems involved, or are due to indirect effects mediated by a chain of events leading to this result. The most common mechanisms which underlie receptors' modulation relate to changes in receptor affinity, variations in the ions permeability, and/or in the transduction signaling pathways, as well as changes in receptor trafficking. The interaction among co-expressed receptors may modulate neurotransmission function via several receptor systems (Raiteri et al., 1992; Pittaluga et al., 2000, 2005; Marchi and Grilli, 2010; Cong et al., 2011; Musante et al., 2011; Delille et al., 2013).
It is known that nAChRs coexist with glutamatergic $\alpha$-amino3-hydroxy-5-methyl-4-isoxazolepropionic acid (AMPA) and $\mathrm{N}$ methyl-D-aspartate (NMDA) receptors on several neurons and particularly at nerve endings level (Marchi and Grilli, 2010; Marchi et al., 2014; Marks et al., 2014). The present review takes into account this new aspect of neuronal nicotinic activity with particular regard to the effects of nicotine on indirect modulation of glutamate and DA release. Of course, this particular effect of nicotine that occurs at the level of some presynaptic nerve terminals could even concern receptors present at the postsynaptic level thus providing a possible broader look at the action of nicotine in the brain.

\section{Isolated Nerve Terminals in Superfusion: An in vitro Model for Studying Functional Interaction between Presynaptic Receptors}

Isolated nerve endings (synaptosomes) are subcellular particles of functionally active nerve tissue, which can be obtained easily by means of a special homogenization of brain tissues (Gray and Whittaker, 1962). These particles can be purified from the other components of the homogenate by centrifugation in a density gradient (Nagy and Delgado-Escueta, 1984; Dunkley et al., 2008). Synaptosomes have been characterized both morphologically and biochemically (Taupin et al., 1994; Feligioni et al., 2006). Morphologically, they resemble as small rounded pockets of 1-2 microns in diameter. They retain on the neuronal membrane structures present in the nerve ending in vivo (i.e., receptors, carriers, etc.). Therefore, when incubated in suitable physiological solutions, synaptosomes are capable of performing the neurochemical activities characteristic of the nerve terminals from which they originate. An important point is that synaptosomes are derived from neurons that were formed, developed, and differentiated in their natural environment, the living brain, allowing a reliable analysis of the events that occurs in CNS. More specifically, the synaptosomes' neuronal origin as well as the lack of the neuronal/glial network that usually prime the physiological plasticity of neurons reduce eventual sources of bias for downstream analyses. This aspect must be taken into consideration when using other in vitro experimental models, such as primary neuronal cultures, which often have glial networks still present to improve culture conditions. The use of the superfusion technique in which synaptosomes are layered in a monolayer on microporous filters and up-down superfused with physiological solutions, has allowed us to overcome most of the problems associated with studies of neurotransmitter release with nerve endings in incubation (Raiteri et al., 1974). Under these experimental conditions, the superfusion medium removes the neurotransmitters released before they can interact and activate structures such as receptors or carriers present on the nerve endings, thereby excluding any possible indirect effects. Accordingly, under these experimental conditions the targets on the synaptosomal membranes such as receptors and/or carriers can be selectively activated by ligands present in the perfusion medium. Therefore, any effect on the release of a neurotransmitter 
may be attributed exclusively to an action on targets present on the nerve ending that specifically releases that neurotransmitter. In fact, neurotransmitter release can be triggered not only by depolarizing substances $(\mathrm{KCl}$, veratrine, etc.), but also by drugs activating receptors present on the nerve terminals and which are stimulated by nicotinic and glutamatergic agonists. For all these reasons, this well-known preparation (Gray and Whittaker, 1962; Raiteri et al., 1974) remains an excellent model for studying molecular and subcellular mechanisms of neurotransmission.

Moreover, using synaptosomes in perfusion to study receptors that modulate the release of neurotransmitters, supplemented and integrated with data obtained by molecular biology, immunocytochemistry techniques, and by the assessment of receptor trafficking, enables us to obtain novel information. Indeed, we can provide relevant and specific information such as: (a) the direct and unequivocal localization of native receptors on the nerve terminal releasing the neurotransmitter under investigation; (b) the identification of the receptors function and the characterization of their transduction mechanisms; (c) the pharmacological characterization of these receptors and the mechanism of action of novel molecules acting on these sites [i.e., evaluation of the affinity and potency of agonist and antagonist drugs $\left.\left(\mathrm{EC}_{50}, \mathrm{IC}_{50}, \mathrm{pA}_{2}\right)\right]$; and (d) the co-localization of different receptors on the same nerve endings and the presence of a synergistic or antagonistic interactions between them.

This approach can be also used for studying desensitization, trafficking of receptors, changes in their subunits structures, evaluation of positive or negative allosteric drugs, ex vivo assessment of receptor function after chronic treatment with drugs, changes in the environment or during development and aging (enriched environment, stress, etc.), and the neurotransmitters release from human tissue (Russo et al., 1993; Risso et al., 2004; Grilli et al., 2005, 2009a,b; Pittaluga et al., 2007; Musante et al., 2008; Summa et al., 2011; Marchi et al., 2012; Marrocco et al., 2014; Merega et al., 2014).

\section{Pre-Treatment with Nicotinic Agonists Modifies the Function of Glutamatergic NMDA Receptors Present on Nerve Terminals}

The presence of the NMDA receptors (NMDARs) on neuronal membranes is quite important since it is connected to various plastic events of the synapse. Although NMDARs are not mobile like AMPARs, they also migrate from the endoplasmic reticulum to the membrane like all membrane proteins (Groc and Choquet, 2006; Pittaluga et al., 2007). It is therefore possible that they also undergo changes with regard to their presence at the level of neuronal membranes, and this may affect their function and modulatory activity. Several factors may trigger this receptor trafficking, including different stimuli derived by activation of receptors (Pittaluga et al., 2004, 2007; Summa et al., 2011). The specific interaction between the nicotinic cholinergic systems and the function of glutamatergic neurotransmission is a remarkable example.
A short pre-treatment of synaptosomes with nicotine (10 min) decreased the NMDA-induced DA release from nucleus accumbens (NAc) nerve terminals (Figure 1; Salamone et al., 2014). Since the nicotine pre-treatment failed to modify the 4aminopyridine-induced DA overflow, changes in the exocytotic machinery of release do not account for nicotine-induced modifications of the NMDARs function (Salamone et al., 2014). These findings unequivocally demonstrate that both nAChRs and NMDARs are functionally located on the same nerve terminals where they also elicit calcium-dependent neurotransmitter release. Notably, results obtained by studying the calcium availability in individual nerve endings indicate that the pre-treatment with nicotine produced a significant decrease of the calcium transients evoked by the activation of NMDARs (Salamone et al., 2014).

Glutamate N2B subunits (GluN2B) are critical component of presynaptic NMDARs located on NAc DA nerve endings (Salamone et al., 2014). These subunits can undergo phosphorylation and their stoichiometry plays an essential role in the regulation of NMDAR channel properties (Akashi et al., 2009; Murphy et al., 2014), leaving open the possibility that the altered responses of NMDARs might involve either a rearrangement of subunits assembly or a modification of the phosphorylation of NMDARs. Alternatively, a change in NMDARs trafficking typified by a reduction of the number of NMDARs present at the plasma membrane level of DA nerve endings may also occur. Interestingly, this latter hypothesis has been clearly favored since biotinylation studies unveiled a reduced density of GluN2B subunits in NAc synaptosomal plasma membranes following nicotine pre-treatment (Salamone et al., 2014). Altogether, these findings demonstrate a functional interaction, within the same individual dopaminergic terminal, between $\mathrm{nAChR}$ and NR2B-containing NMDARs, through an ability of nicotinic agents to induce endocytosis of these latter receptors (Figure 1; Salamone et al., 2014).

With regard to the possible role of specific subtypes of $n A C h R$ involved in this mechanism, we must remember that different $\mathrm{nAChR}$ subtypes including $\alpha$-conotoxin-sensitive $(\alpha 6 \beta 2 \beta 3$, $\alpha 4 \alpha 6 \beta 2 \beta 3, \alpha 6 \beta 2$, and $\alpha 4 \alpha 6 \beta 2)$ and $\alpha$-conotoxin- insensitive $(\alpha 4 \beta 2$ and $\alpha 4 \alpha 5 \beta 2$ ) subtypes mediate nicotine-evoked DA release from striatal synaptosomes (Kaiser et al., 1998; Salminen et al., 2007). Since the inhibitory effect of nicotine pre-treatment was partially counteracted by $\alpha$ conotoxin MII, it is likely that nAChRs containing the $\alpha 6$ subunit, which is abundant in the NAc (Exley et al., 2008; Hendrickson and Tapper, 2014, and references therein) are also involved. The pre-treatment (10 min) with 5IA85380, a $\alpha 4 / \alpha 6$ nicotine agonist, further confirms this hypothesis. Interestingly, preclinical evidence supports the importance of mesolimbic $\alpha 6$ containing nAChRs in the nicotine addiction (Exley et al., 2008; Brunzell, 2012). Conversely, $\alpha 7-n A C h R$ subtypes do not appear to be involved in any kind of interaction with glutamatergic receptors in NAc DA terminals as heralded also by the absence of effects after the pre-treatment $(10 \mathrm{~min}$ ) with choline (Salamone et al., 2014).

However, in other neurotransmitter systems it seems that even $\alpha 7-n A C h R s$ might be implicated in a cross-talk between nicotinic and glutamatergic receptors. A recent report focusing on glutamatergic synapses indicated that $\alpha 7-n A C h R s$ might enhance 


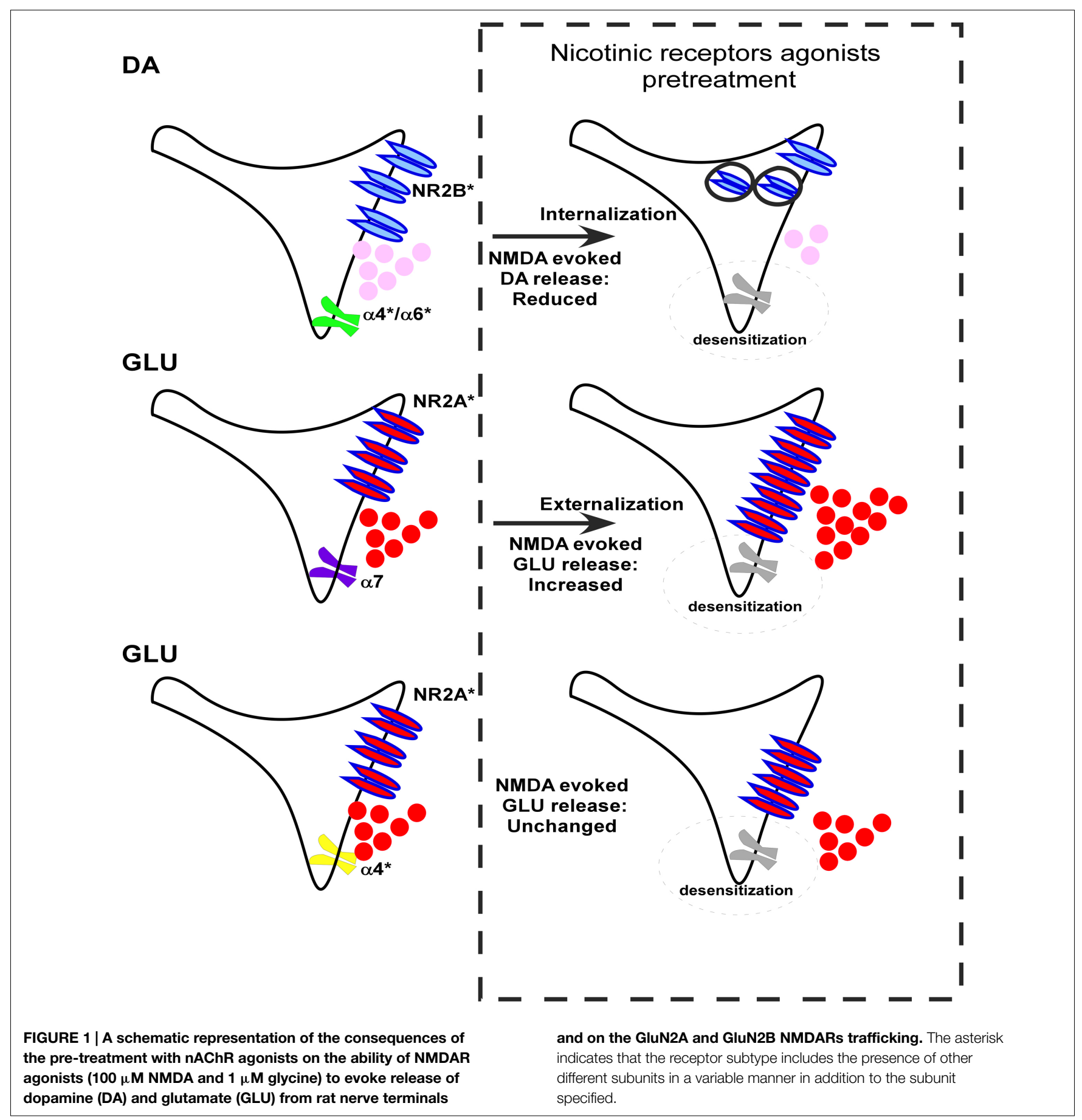

the presynaptic surface expression of NMDARs leading to an increased glutamate release during early synaptic development (Lin et al., 2010). Interestingly, the NMDARs modulating glutamate release are apparently different from those modulating the release of other neurotransmitters in several brain areas (Pittaluga et al., 2001). It can be therefore suggested that the interaction between nAChRs and NMDARs could be differently organized in different types of nerve terminals, possibly depending on the different $\mathrm{nAChRs}$ and NMDARs subtypes involved. In support of this hypothesis, a pre-treatment $(10 \mathrm{~min})$ of NAc glutamatergic synaptosomes with nicotine or the $\alpha 7$ agonists choline caused a significant increase of the NMDA-evoked overflow of $\left[{ }^{3} \mathrm{H}\right] \mathrm{D}$-ASP while, conversely, the pre-treatment $(10 \mathrm{~min})$ of glutamatergic nerve endings with the $\alpha 4 / \alpha 6$ nicotinic agonist 5IA85380 was ineffective (Figure 1; Zappettini et al., 2014).

The possibility that nicotinic and NMDA receptors coexist and may interact on glutamatergic terminals is not surprising. Indeed, the presence of $\alpha 4 \beta 2$ and $\alpha 7$ AChRs on glutamatergic 
nerve endings is well documented in several brain regions (for a review, see Vizi and Lendvai, 1999) as well as the presence of NMDA receptors that modulate the release of glutamate (Luccini et al., 2007; Zappettini et al., 2010; Musante et al., 2011). The pharmacological characterization indicates that GluN2A subunits participate in the expression of these presynaptic NMDARs located on glutamatergic nerve endings, which therefore differ from those containing GluN2B subunits present on NAc DA nerve terminals (Zappettini et al., 2014). Thus, at least some of these NMDARs are functionally expressed and co-localized both with $\alpha 7$ nAChRs with whom they can functionally interact. Furthermore, the pre-treatment with nicotine or choline produced a significant increase of the calcium transients evoked by the activation of NMDARs; this event occurred only in glutamatergic nerve endings (Zappettini et al., 2014). This finding is therefore consistent with the data summarized above, and supports the hypothesis that the facilitation of the NMDA-evoked overflow could be due to an increase of the synaptosomal calcium transients (Zappettini et al., 2014), yet considering that calcium influx can influence directly neurotransmitter release. It is also important to recall that NMDARs coexist also with $\alpha 4 \beta 2 \mathrm{nAChR}$ subtypes in glutamatergic terminals, but in this case without any functional interaction among them (Figure 1; Zappettini et al., 2014). Interestingly, biotinylation studies have shown an increased density of GluN2A subunits in synaptosomal plasma membranes following nicotine and choline pre-treatment but no changes after preincubation with 5IA85380 (Zappettini et al., 2014). These findings demonstrate the ability of $\alpha 7$ nicotinic but not of $\alpha 4 \beta 2$ agonists, to induce an increase in the trafficking of GluN2A-containing NMDARs and consequently an increase of the functional responses.

In conclusion, the nicotine-evoked internalization of NMDARs present on DA nerve endings appears not to depend upon the activation of one particular subtype of $\mathrm{nAChR}$, but rather on the characteristics of the NMDARs coexisting with nAChRs on the same nerve terminal. Conversely, the increase of the response of the NMDARs present on the glutamatergic nerve endings after nicotine pre-treatment might depend on two concomitant factors: (a) the activation of a specific nAChR subtype $(\alpha 7)$, and (b) the coexistence of these receptors with NMDARs which contain the GluN2A subunit (Figure 1; Salamone et al., 2014; Zappettini et al., 2014).

\section{Pre-Treatment with Nicotinic Agonists Modifies the Function of Presynaptic Glutamatergic AMPA Receptors}

Alpha-amino-3-hydroxy-5-methyl-4-isoxazolepropionic acid are largely responsible for the rapid post-synaptic response to glutamate, are widely present in the CNS, and are differently distributed both at the post- and presynaptic level. Accordingly, a significant percentage of dopaminergic terminals present in the NAc possess GluA2 receptor subunits as demonstrated by functional and immuno-cytochemistry studies (Grilli et al., 2012). The AMPARs are highly dynamic in their distribution and can cycle rapidly among the synaptic membrane and intracellular compartments (Henley, 2003; Pittaluga et al., 2006). As a confirmation of this mobility, even within the nerve endings, the pre-treatment (10 min) of NAc synaptosomes with nicotine caused a significant reduction of the AMPA-evoked overflow of DA from NAc nerve terminals. Accordingly, the loss of function of AMPARs in nicotine pre-treated synaptosomes was accompanied by a decreased insertion of GluA2 subunit proteins in the presynaptic component of synaptosomal plasma membranes in the nicotine-treated nerve endings (Figure 2; Grilli et al., 2012). Interestingly, the decrease of the AMPA-induced DA overflow in nicotine-pre-treated NAc dopaminergic terminals was significantly counteracted in synaptosomes containing entrapped pep2-SVKI, a peptide known to compete for the binding of GluA2 subunit to scaffolding proteins involved in AMPARs internalization. Thus, it is likely that nicotine pre-treatment could favor the internalization of GluA2containing AMPARs located on dopaminergic NAc terminals. This event, however, seems to occur in a selected population of nerve terminals, since AMPA-induced releasing effect was halved, but not abolished, by nicotine (Figure 2; Grilli et al., 2012).

Interestingly, the nicotine-induced changes to releaseregulating presynaptic AMPARs was not limited to DA terminals, but could also be observed when studying the AMPA-evoked release of noradrenaline from hippocampal nerve terminals (Grilli et al., 2012). However, nicotine-induced adaptation of AMPARs does not represent a generalized event that occurs in all nerve endings. Indeed, the pre-treatment of NAc synaptosomes to nicotine failed to cause significant changes of the AMPAevoked overflow of $\gamma$-aminobutyric acid (GABA) and ACh from these terminals (Figure 2; Grilli et al., 2012). The most likely explanation for the selective endocytosis of AMPARs that modulates DA and NA release can be the presence of similar cyclothiazide-sensitive AMPA receptors endowed with GluA2 subunits, which are susceptible to the internalization (Pittaluga et al., 1999, 2006; Ghersi et al., 2003; Grilli et al., 2012). Conversely, the AMPA receptors controlling acetylcholine and GABA release are cyclothiazide-insensitive and did not undergo a GRIP/ABP/ PICK1-dependent constitutive trafficking (Pittaluga et al., 1997, 2006).

Due to the very heterogeneous presence of nAChRs on dopaminergic and noradrenergic nerve endings (Sershen et al., 1997; Zoli et al., 2002; Kennett et al., 2012; Pistillo et al., 2015, and references therein), it is very difficult to correlate specific $\mathrm{nAChR}$ subtypes with the internalization of AMPA receptors. In any case, the involvement of $\alpha 7 \mathrm{nAChR}$ subtype can be excluded based on the lack of effect of the specific $\alpha 7$ agonist choline due to the absence of this nAChR subtype on both dopaminergic and noradrenergic terminals (Clarke and Reuben, 1996; Kaiser and Wonnacott, 2000).

\section{Conclusions}

As summarized above, studies have shown that in the rat NAc the function of specific GluRs present on the dopaminergic and glutamatergic nerve terminals can be negatively or positively regulated in response to a brief activation of nAChRs (Grilli et al., 2012; Salamone et al., 2014; Zappettini et al., 2014). The effect occurs rapidly and seems to be entirely caused by the trafficking 


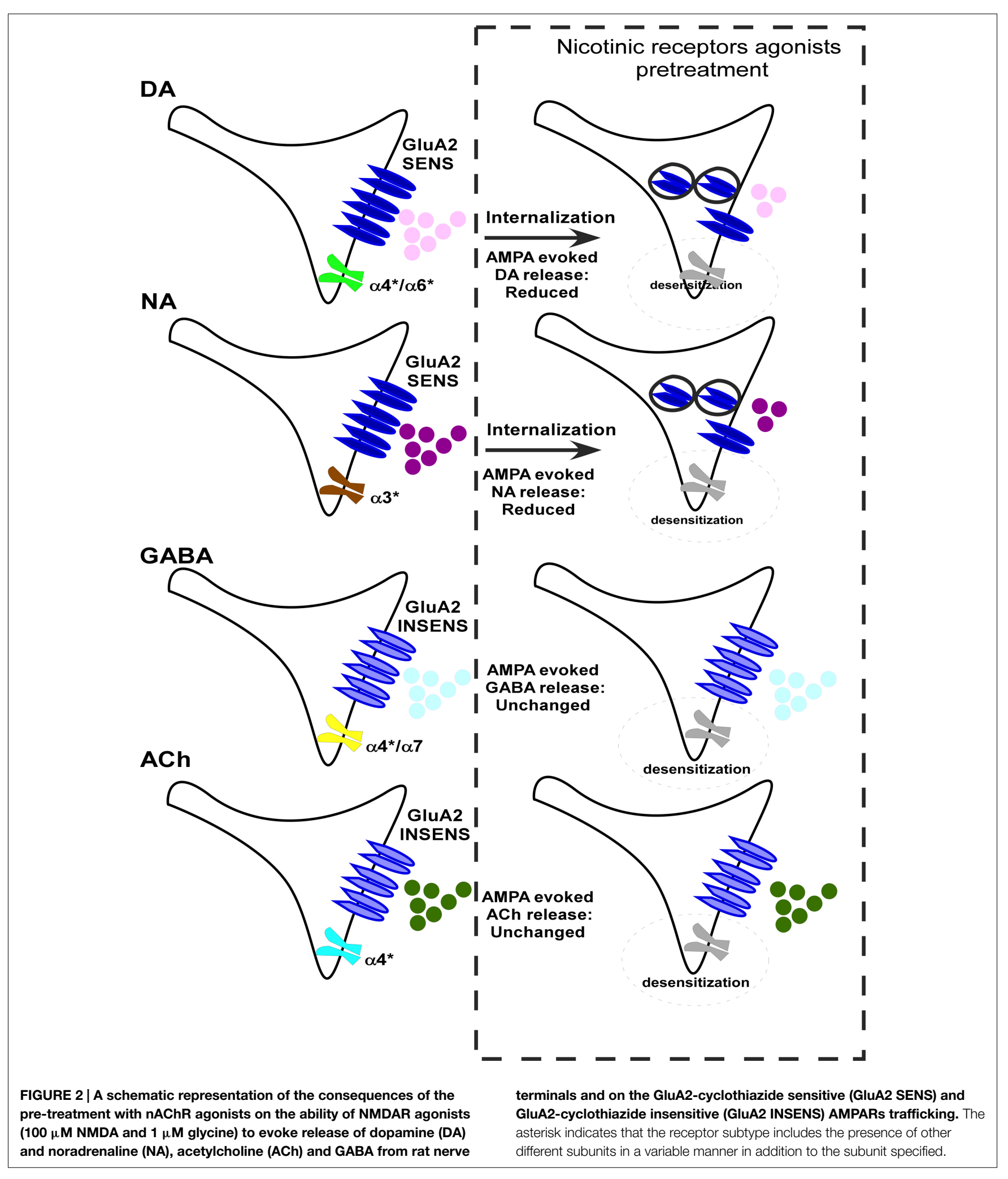

of AMPA and NMDA receptors. However, this takes place only in certain nerve terminals and after the activation of either $\alpha 7$ or non- $\alpha 7 \mathrm{nAChR}$ subtypes. It is quite important to note that these effects occur at very low concentrations of nicotine (Salamone et al., 2014). Interestingly, these nicotine levels, which alone are unable to evoke release of neurotransmitters, are compatible with the nicotine concentrations present in the venous plasma of heavy smokers (Benowitz, 1988; for a review, see Matta et al., 2007) or 
even of people under nicotine replacement therapy for smoking cessation.

Moreover, it is important to note that nicotine at these concentrations switches its typical stimulatory effects on DA release into an inhibitory effect; this phenomenon could be a fine-tuning of synaptic signals in response to the increased glutamatergic activity mediated by the upregulation of NR2A-containing NMDARs.

The dynamic control by cholinergic nicotinic system of glutamatergic NMDA and AMPA receptors might therefore represent an important neuronal adaptation associated with nicotine administration. If and how this event may have a functional relevance or may play an important role in the clarification of the mechanism of action of nicotine may be worth investigating in the future. Indeed, the understanding of the role of these nicotineinduced functional changes might open new and interesting perspectives in terms of explaining the mechanisms that underlie some of the effects of nicotine addiction and the development of new drugs for smoking cessation.

It is rather complicated to contextualize the interaction of these two neurotransmitter systems mainly because of two reasons: (1) many neurons are able to interact to each other without making synapses. Recently, functional and morphological evidence has clearly demonstrated that most neurotransmitters can diffuse once released and achieve distant targets in the CNS, provided that the target neurons are equipped with receptors (Lendvai and Vizi, 2008); and (2) nACh, AMPA and NMDA receptors are located both synaptically and extrasynaptically (Groc et al., 2009, and references therein; Petralia et al., 2009). In fact, it has been proposed that GluNR2B-NMDARs are mostly extrasynaptically while GluNR2A-NMDARs seem to be located mostly at synaptic level (Groc et al., 2009, and references therein, Petralia et al., 2009; Sanz-Clemente et al., 2013). Therefore, further investigation on the interaction of these two neurotransmitter systems at the nerve terminal level will hopefully shed light on the role that this mechanism could play both in normal physiology as well as in nicotine dependence. Of relevance, Pistillo et al. (2015) reviewed the molecular, functional, and behavioral mechanisms involved in the nicotine-induced effects in the mesocorticolimbic system, and discussed the dopaminergic and glutamatergic circuits within these CNS regions.

The cellular and molecular mechanisms that regulate the presence of NMDA and AMPA receptors on neurons in the CNS have been thoroughly investigated over the last decades. There are several identified mechanisms that can explain the GluRs trafficking and the consequent impact that these events might have on the receptors responses (Zhou et al., 2015). NMDAR surface trafficking is subunit dependent being GluN2A-NMDARs less mobile and more retained within synapse than GluN2BNMDARs. Approximately $30-40 \%$ of surface GluN2B-NMDAR are mobile and the average of synaptic residency time appears to be minutes (Tovar and Westbrook, 2002; Bard and Groc, 2011, and references therein).

It has been recently reported that NMDARs may also diffuse on the surface of neurons in a highly dynamic process (Groc et al., 2009). The mobility of presynaptic receptors on the neuron surface might therefore generate different functional responses (Gomez-Varela and Berg, 2013). How and through the activation of which mechanisms nicotine could control the surface dynamics of GluRs at the nerve terminal levels is at present not easy to explain. However, although we cannot establish whether the pretreatment with nicotine is able to influence the rapid and lateral redistribution of GluRs at the membrane level, we can reasonable assume that the changes in the functional response of GluRs might to be linked to their trafficking from the cytoplasm to the surface of the terminal. Additional investigation is needed to clarify which mechanism(s) regulating the trafficking of AMPA and NMDA receptors so far reported is/are triggered or inhibited by the pretreatment with nicotine. In fact, a physical interaction between $\alpha 7$ nAChR and the GluNR2A- NMDAR has been recently reported (Li et al., 2013). Therefore, the possibility that the pre-treatment with $\alpha 7$ agonists might upregulate the $\alpha 7 \mathrm{nAChR} / \mathrm{NMDAR}$ complex and consequently enhance GluNR2A-NMDARs cell surface expression has to be also considered.

Several lines of evidence in laboratory animals and preclinical studies demonstrate that different glutamatergic receptors are critically involved in several nicotine-mediated effects (Vizi and Lendvai, 1999; Laviolette and Van Der Kooy, 2004; Dani and Bertrand, 2007; Markou, 2008; Timofeeva and Levin, 2011; Zhong et al., 2014). This dynamic control by cholinergic nicotinic system of both NMDA and AMPA receptors might therefore be crucial for many forms of synaptic plasticity including events linked to nicotine dependence. Moreover, this event should be also relevant to understand the interplay between nAChRs and NMDARs in neurodegenerative diseases. Epidemiological studies have identified a negative correlation between smoking and the development of neurodegenerative disorders such as Parkinson's disease, and in some studies, Alzheimer's disease (Picciotto and Zoli, 2008). These findings have been attributed to the ability of nicotine to act as a neuroprotective agent. Indeed, a certain number of studies demonstrated that nicotine can protect against neuronal death in vitro and in vivo (Dajas-Bailador et al., 2000; Jonnala and Buccafusco, 2001; Quik et al., 2007; Picciotto and Zoli, 2008; Shimohama, 2009; Akaike et al., 2010; Bordia et al., 2015). However, it is also very important to recall that several studies have reported that nicotine is neurotoxic to several neuronal subtypes, including immature neurons (Trauth et al., 2000; Laudenbach et al., 2002).

Although relevant, our findings are insufficient to support a strict correlation among these new nicotine-induced plastic modifications at nerve terminals, and the events that underlie nicotine addiction as well as nicotine-induced promnesic and neuroprotectant actions. Nevertheless, the working hypothesis is promising and surely deserves future investigation aimed at highlighting the roles that nicotine /glutamate receptor functional cross talk play in the mechanism of synaptic plasticity induced by nicotine.

\section{Acknowledgments}

We wish to thank Maura Agate and Silvia E. Smith, Ph.D. (University of Utah, School of Medicine) for editorial assistance. MM and AMP would like to express deep gratitude to Prof. Maurizio Raiteri, a pioneer in this field, for having long time ago introduced them to the exciting world of "synaptosomes in superfusion" and always inspired and encouraged their research. 


\section{References}

Akaike, A., Takada-Takatori, Y., Kume, T., and Izumi, Y. (2010). Mechanisms of neuroprotective effects of nicotine and acetylcholinesterase inhibitors: role of $\alpha 4$ and $\alpha 7$ receptors in neuroprotection. J. Mol. Neurosci. 40, 211-216. doi: 10.1007/ s12031-009-9236-1

Akashi, K., Kakizaki, T., Kamiya, H., Fukaya, M., Yamasaki, M., Abe, M., et al. (2009). NMDA receptor GluN2B (GluRe2/NR2B) subunit is crucial for channel function, postsynaptic macromolecular organization, and actin cytoskeleton at hippocampal CA3 synapses. J. Neurosci. 29, 10869-10882. doi: 10.1523/ JNEUROSCI.5531-08.2009

Arqueros, L., Naquira, D., and Zunino, E. (1978). Nicotine-induced release of catecholamines from rat hippocampus and striatum. Biochem. Pharmacol. 27, 2667-2674. doi: 10.1016/0006-2952(78)90040-0

Bard, L., and Groc, L. (2011). Glutamate receptor dynamics and protein interaction: lessons from the NMDA receptor. Mol. Cell. Neurosci. 48, 298-307. doi: 10.1016/j.mcn.2011.05.009

Benowitz, N. L. (1988). Drug therapy. Pharmacologic aspects of cigarette smoking and nicotine addiction. New Engl. J. Med. 319, 1318-1330. doi: 10.1056/ NEJM198811173192005

Bertrand, D., Ballivet, M., and Rungger, D. (1990). Activation and blocking of neuronal nicotinic acetylcholine receptor reconstituted in Xenopus oocytes. Proc. Natl. Acad. Sci. U.S.A. 87, 1993-1997. doi: 10.1073/pnas.87.5.1993

Bordia, T., McGregor, M., Papke, R. L., Decker, M. W., McIntosh, J. M., and Quik, M. (2015). The $\alpha 7$ nicotinic receptor agonist ABT-107 protects against nigrostriatal damage in rats with unilateral 6-hydroxydopamine lesions. Exp. Neurol. 263, 277-284. doi: 10.1016/j.expneurol.2014.09.015

Brunzell, D. H. (2012). Preclinical evidence that activation of mesolimbic $\alpha 6$ subunit containing nicotinic acetylcholine receptors supports nicotine addiction phenotype. Nicotine Tob. Res. 14, 1258-1269. doi: 10.1093/ntr/nts089

Buisson, B., Gopalakrishnan, M., Arneric, S. P., Sullivan, J. P., and Bertrand, D. (1996). Human $\alpha 4 \beta 2$ neuronal nicotinic acetylcholine receptor in HEK 293 cells: a patch-clamp study. J. Neurosci. 16, 7880-7891.

Clarke, P. B., and Reuben, M. (1996). Release of [3H]-noradrenaline from rat hippocampal synaptosomes by nicotine: mediation by different nicotinic receptor subtypes from striatal [3H]-dopamine release. Br. J. Pharmacol. 117, 595-606. doi: 10.1111/j.1476-5381.1996.tb15232.x

Cong, D., Tang, Z., Li, L., Huang, Y., Wang, J., and Chen, L. (2011). Cross-talk between NMDA and GABAA receptors in cultured neurons of the rat inferior colliculus. Sci. China Life Sci. 54, 560-566. doi: 10.1007/s11427-011-4178-6

Dajas-Bailador, F. A., Lima, P. A., and Wonnacott, S. (2000). The $\alpha 7$ nicotinic acetylcholine receptor subtype mediates nicotine protection against NMDA excitotoxicity in primary hippocampal cultures through a $\mathrm{Ca}^{2+}$ dependent mechanism. Neuropharmacology 39, 2799-2807. doi: 10.1016/S0028-3908(00) 00127-1

Dajas-Bailador, F., and Wonnacott, S. (2004). Nicotinic acetylcholine receptors and the regulation of neuronal signalling. Trends Pharmacol. Sci. 25, 317-324. doi: 10.1016/j.tips.2004.04.006

Dani, J. A., and Bertrand, D. (2007). Nicotinic acetylcholine receptors and nicotinic cholinergic mechanisms of the central nervous system. Annu. Rev. Pharmacol. Toxicol. 47, 699-729. doi: 10.1146/annurev.pharmtox.47.120505.105214

Delille, H. K., Mezler, M., and Marek, G. J. (2013). The two faces of the pharmacological interaction of mGlu2 and 5-HT2A e relevance of receptor heterocomplexes and interaction through functional brain pathways. Neuropharmacology 70, 296-305. doi: 10.1016/j.neuropharm.2013.02.005

Dickinson, J. A., Kew, J. N., and Wonnacott, S. (2008). Presynaptic $\alpha 7$-and $\beta 2$ containing nicotinic acetylcholine receptors modulate excitatory amino acid release from rat prefrontal cortex nerve terminals via distinct cellular mechanisms. Mol. Pharmacol. 74, 348-359. doi: 10.1124/mol.108.046623

Dunkley, P. R., Jarvie, P. E., and Robinson, P. J. (2008). A rapid Percoll gradient procedure for preparation of synaptosomes. Nat. Protoc. 3, 1718-1728. doi: 10.1038/nprot.2008.171

Exley, R., Clements, M. A., Hartung, H., McIntosh, J. M., and Cragg, S. J. (2008). $\alpha 6$-containing nicotinic acetylcholine receptors dominate the nicotine control of dopamine neurotransmission in nucleus accumbens. Neuropsychopharmacology 33, 2158-2166. doi: 10.1038/sj.npp.1301617

Feligioni, M., Holman, D., Haglerod, C., Davanger, S., and Henley, J. M. (2006). Ultrastructural localisation and differential agonist-induced regulation of AMPA and kainate receptors present at the presynaptic active zone and postsynaptic density. J. Neurochem. 99, 549-560. doi: 10.1111/j.1471-4159.2006. 04087.x

Ghersi, C., Bonfanti, A., Manzari, B., Feligioni, M., Raiteri, M., and Pittaluga, A. (2003). Pharmacological heterogeneity of release-regulating presynaptic AMPA/kainite receptors in the rat brain: study with receptor antagonists. Neurochem. Int. 42, 283-292. doi: 10.1016/S0197-0186(02)00129-8

Gomez-Varela, D., and Berg, D. K. (2013). Lateral mobility of presynaptic $\alpha 7$ containing nicotinic receptors and its relevance for glutamate release. J. Neurosci. 33, 17062-17071. doi: 10.1523/JNEUROSCI.1482-13.2013

Gray, E. G., and Whittaker, V. P. (1962). The isolation of nerve endings from brain: an electron-microscopic study of cell fragments derived by homogenization and centrifugation. J. Anat. 96, 79-88.

Grilli, M., Parodi, M., Raiteri, M., and Marchi, M. (2005). Chronic nicotine differentially affects the function of nicotinic receptor subtypes regulating neurotransmitter release. J. Neurochem. 93, 1353-1360. doi: 10.1111/j.14714159.2005.03126.x

Grilli, M., Patti, L., Robino, F., Zappettini, S., Raiteri, M., and Marchi, M. (2008). Release-enhancing pre-synaptic muscarinic and nicotinic receptors co-exist and interact on dopaminergic nerve endings of rat nucleus accumbens. J. Neurochem. 105, 2205-2213. doi: 10.1111/j.1471-4159.2008.05307.x

Grilli, M., Summa, M., Salamone, A., Olivero, G., Zappettini, S., Di Prisco, S., et al. (2012). In vitro exposure to nicotine induces endocytosis of presynaptic AMPA receptors modulating dopamine release in rat nucleus accumbens nerve terminals. Neuropharmacology 63, 916-926. doi: 10.1016/j.neuropharm.2012.06.049

Grilli, M., Zappettini, S., Zanardi, A., Lagomarsino, F., Pittaluga, A., Zoli, M., et al. (2009a). Exposure to an enriched environment selectively increases the functional response of the pre-synaptic NMDA receptors which modulate noradrenaline release in mouse hippocampus. J. Neurochem. 110, 1598-1606. doi: 10.1111/j.1471-4159.2009.06265.x

Grilli, M., Zappettini, S., Zoli, M., and Marchi, M. (2009b). Pre-synaptic nicotinic and D2 receptors functionally interact on dopaminergic nerve endings of rat and mouse nucleus accumbens. J. Neurochem. 108, 1507-1514. doi: 10.1111/j.14714159.2009.05933.x

Groc, L., Bard, L., and Choquet, D. (2009). Surface trafficking of N-methyl-Daspartate receptors: physiological and pathological perspectives. Neuroscience 158, 4-18. doi: 10.1016/j.neuroscience.2008.05.029

Groc, L., and Choquet, D. (2006). AMPA and NMDA glutamate receptor trafficking: multiple roads for reaching and leaving the synapse. Cell Tissue Res. 26, 423-438. doi: 10.1007/s00441-006-0254-9

Hendrickson, L. M., and Tapper, A. R. (2014). "Neuronal nicotinic acetylcholine receptors in reward and addiction," in Nicotinic Receptors, ed. R. A. J. Lester (New York: Springer), 307-325. doi: 10.1007/978-1-4939-1167-7_15

Henley, J. M. (2003). Proteins interactions implicated in AMPA receptor trafficking: a clear destination and an improving route map. Neurosci. Res. 45, 243-254. doi: 10.1016/S0168-0102(02)00229-8

Jones, I. W., and Wonnacott, S. (2005). Why doesn't nicotinic ACh receptor immunoreactivity knock out? Trends Neurosci. 28, 343-345. doi: 10.1016/j.tins. 2005.04.010

Jonnala, R. R., and Buccafusco, J. J. (2001). Relationship between the increased cell surface $\alpha 7$ nicotinic receptor expression and neuroprotection induced by several nicotinic receptor agonists. J. Neurosci. Res. 66, 565-572. doi: 10.1002/jnr.10022

Kaiser, S. A., Soliakov, L., Harvey, S. C., Luetje, C. W., and Wonnacott, S. (1998). Differential inhibition by $\alpha$-conotoxin-MII of the nicotinic stimulation of $[3 \mathrm{H}]$ dopamine release from rat striatal synaptosomes and slices. J. Neurochem. 70, 1069-1076. doi: 10.1046/j.1471-4159.1998.70031069.x

Kaiser, S., and Wonnacott, S. (2000). $\alpha$-bungarotoxin-sensitive nicotinic receptors indirectly modulate $[3 \mathrm{H}]$ dopamine release in rat striatal slices via glutamate release. Mol. Pharmacol. 58, 312-318. doi: 10.1124/mol.58.2.312

Kennett, A., Heal, D. J., and Wonnacott, S. (2012). Pharmacological differences between rat frontal cortex and hippocampus in the nicotinic modulation of noradrenaline release implicate distinct receptor subtypes. Nicotine Tob. Res. 14, 1339-1345. doi: 10.1093/ntr/nts128

Laudenbach, V., Medja, F., Zoli, M., Rossi, F. M., Evrard, P., Changeux, J. P., et al. (2002). Selective activation of central subtypes of the nicotinic acetylcholine receptor has opposite effects on neonatal excitotoxic brain injuries. FASEB J. 16, 423-425. doi: 10.1096/fj.01-0532fje

Laviolette, S. R., and Van Der Kooy, D. (2004). The neurobiology of nicotine addiction: bridging the gap from molecules to behaviour. Nature Rev. Neurosci. 5, 55-65. doi: 10.1038/nrn1298 
Lendvai, B., and Vizi, E. S. (2008). Nonsynaptic chemical transmission through nicotinic acetylcholine receptors. Physiol. Rev. 88, 333-349. doi: 10.1152/physrev.00040.2006

Li, S., Nai, Q., Lipina, T. V., Roder, J. C., and Liu, F. (2013). $\alpha 7$ nAchR/NMDAR coupling affects NMDAR function and object recognition. Mol. Brain 6, 58. doi: 10.1186/1756-6606-6-58

Lin, H., Vicini, S., Hsu, F. C., Doshi, S., Takano, H., Coulter, D. A., et al. (2010). Axonal $\alpha 7$ nicotinic ACh receptors modulate presynaptic NMDA receptor expression and structural plasticity of glutamatergic presynaptic boutons. Proc. Natl. Acad. Sci. U.S.A. 107, 16661-16666. doi: 10.1073/pnas.1007397107

Luccini, E., Musante, V., Neri, E., Brambilla Bas., M., Severi, P., Raiteri, M., et al. (2007). Functional interactions between presynaptic NMDA receptors and metabotropic glutamate receptors co-expressed on rat and human noradrenergic terminals. Br. J. Pharmacol. 151, 1087-1094. doi: 10.1038/sj.bjp.0707280

Marchi, M., and Grilli, M. (2010). Presynaptic nicotinic receptors modulating neurotransmitter release in the Central Nervous System: functional interactions with other coexisting receptors. Prog. Neurobiol. 92, 105-111. doi: 10.1016/ j.pneurobio.2010.06.004

Marchi, M., Grilli, M., and Pittaluga, A. (2014). A new specific neuronal modulatory effect of nicotine: the functional cross talk between nicotinic and glutamate receptors. Receptor Clin. Invest. 1, 68-71. doi: 10.14800/rci.84

Marchi, M., Risso, F., Viola, C., Cavazzani, P., and Raiteri, M. (2002). Direct evidence that release-stimulating $\alpha 7^{*}$ nicotinic cholinergic receptors are localized on human and rat brain glutamatergic axon terminals. J. Neurochem. 80, 1071-1078. doi: 10.1046/j.0022-3042.2002.00805.x

Marchi, M., Zappettini, S., Olivero, G., Pittaluga, A., and Grilli, M. (2012). Chronic nicotine exposure selectively activates a carrier-mediated release of endogenous glutamate and aspartate from rat hippocampal synaptosomes. Neurochem. Int. 60, 622-630. doi: 10.1016/j.neuint.2012.02.032

Markou, A. (2008). Neurobiology of nicotine dependence. Philos. Trans. R. Soc. Lond. B Biol. Sci. 363, 3159-3168. doi: 10.1098/rstb.2008.0095

Marks, M. J., Grady, S. R., McClure-Begley, T. D., O’Neill, H. C., and Zambrano, C. A. (2014). "Presynaptic nicotinic acetylcholine receptors: subtypes and functions," in Nicotinic Receptors, ed. R. A. J. Lester (New York: Springer), 61-92. doi: 10.1007/978-1-4939-1167-7_4

Marrocco, J., Reynaert, M. L., Gatta, E., Gabriel, C., Mocaër, E., Di Prisco, S., et al. (2014). The effects of antidepressant treatment in prenatally stressed rats support the glutamatergic hypothesis of stress-related disorders. J. Neurosci. 34, 2015-2024. doi: 10.1523/JNEUROSCI.4131-13.2014

Matta, S. G., Balfour, D. J., Benowitz, N. L., Boyd, R. T., Buccafusco, J. J., Caggiula, A. R., et al. (2007). Guidelines on nicotine dose selection for in vivo research. Psychopharmacology 190, 269-319. doi: 10.1007/s00213-006-0441-0

McGehee, D. S., and Role, L. W. (1995). Physiological diversity of nicotinic acetylcholine receptors expressed by vertebrate neurons. Annu. Rev. Physiol. 57, 521-546. doi: 10.1146/annurev.ph.57.030195.002513

Merega, E., Di Prisco, S., Lanfranco, M., Severi, P., and Pittaluga, A. (2014). Complement selectively elicits glutamate release from nerve endings in different regions of mammal central nervous system. J. Neurochem. 129, 473-483. doi: $10.1111 /$ jnc. 12650

Murphy, J. A., Stein, I. S., Lau, C. G., Peixoto, R. T., Aman, T. K., Kaneko, N., et al. (2014). Phosphorylation of Ser1166 on GluN2B by PKA is critical to synaptic NMDA receptor function and $\mathrm{Ca}^{2+}$ signaling in spines. J. Neurosci. 34, 869-879. doi: 10.1523/JNEUROSCI.4538-13.2014

Musante, V., Neri, E., Feligioni, M., Puliti, A., Pedrazzi, M., Conti, V., et al. (2008). Presynaptic mGlu1 and mGlu5 autoreceptors facilitate glutamate exocytosis from mouse cortical nerve endings. Neuropharmacology 55, 474-482. doi: 10.1016/j.neuropharm.2008.06.056

Musante, V., Summa, M., Cunha, R. A., Raiteri, M., and Pittaluga, A. (2011). Presynaptic glycine GlyT1 transporter-NMDA receptor interaction: relevance to NMDA autoreceptor activation in the presence of $\mathrm{Mg}^{2+}$ ions. J. Neurochem. 117, 516-527. doi: 10.1111/j.1471-4159.2011.07223.x

Nagy, A., and Delgado-Escueta, A. V. (1984). Rapid preparation of synaptosomes from mammalian brain using nontoxic isosmotic gradient material (Percoll). J. Neurochem. 43, 1114-1123. doi: 10.1111/j.1471-4159.1984.tb12851.x

Parodi, M., Patti, L., Grilli, M., Raiteri, M., and Marchi, M. (2006). Nicotine has a permissive role on the activation of metabotropic glutamate 5 receptors coexisting with nicotinic receptors on rat hippocampal noradrenergic nerve terminals. Neurochem. Int. 48, 138-143. doi: 10.1016/j.neuint.2005.08. 010
Patti, L., Raiteri, L., Grilli, M., Parodi, M., Raiteri, M., and Marchi, M. (2006). P2X7 receptors exert a permissive role on the activation of release-enhancing presynaptic $\alpha 7$ nicotinic receptors co-existing on rat neocortex glutamatergic terminals. Neuropharmacology 50, 705-713. doi: 10.1016/j.neuropharm.2005.11.016

Petralia, R. S., Al-Hallaq, R. A., and Wenthold, R. J. (2009). “Trafficking and targeting of NMDA receptors," in Biology of the NMDA Receptor, ed. A. M. Van Dongen (Boca Raton, FL: CRC Press), 149-200. doi: 10.1201/9781420044157.ch8

Picciotto, M. R., and Zoli, M. (2008). Neuroprotection via nAChRs: the role of nAChRs in neurodegenerative disorders such as Alzheimer's and Parkinson's disease. Front. Biosci. 13:492-504. doi: 10.2741/2695

Pistillo, F., Clementi, F., Zoli, M., and Gotti, C. (2015). Nicotinic, glutamatergic and dopaminergic synaptic transmission and plasticity in the mesocorticolimbic system: focus on nicotine effects. Prog. Neurobiol. 124, 1-27. doi: 10.1016/ j.pneurobio.2014.10.002

Pittaluga, A., Bonfanti, A., Arvigo, D., and Raiteri, M. (1999). Aniracetam, 1-BCP and cyclothiazide differentially modulate the function of NMDA and AMPA receptors mediating enhancement of noradrenaline release in rat hippocampal slices. Naunyn Schmiedeberg's Arch. Pharmacol. 359, 272-279. doi: 10.1007/ PL00005352

Pittaluga, A., Bonfanti, A., and Raiteri, M. (1997). Differential desensitization of ionotropic non-NMDA receptors having distinct neuronal location and function. Naunyn Schmiedeberg's Arch. Pharmacol. 356, 29-38. doi: 10.1007/ PL00005025

Pittaluga, A., Bonfanti, A., and Raiteri, M. (2000). Somatostatin potentiates NMDA receptor function via activation of $\mathrm{Ins} 3 \mathrm{receptors}$ and $\mathrm{PKC}$ leading to removal of the $\mathrm{Mg}^{2+}$ block without depolarization. Br. J. Pharmacol. 130, 557-566. doi: 10.1038/sj.bjp.0703346

Pittaluga, A., Feligioni, M., Longordo, F., Arvigo, M., and Raiteri, M. (2005). Somatostatin induced activation and up-regulation of $N$-methyl-D-aspartate receptor function: mediation through calmodulin-dependent protein kinase II, phospholipase $\mathrm{C}$, protein kinase $\mathrm{C}$, and tyrosine kinase in hippocampal noradrenergic nerve endings. J. Pharmacol. Exp. Ther. 313, 242-249. doi: 10.1124/ jpet.104.079590

Pittaluga, A., Feligioni, M., Longordo, F., Luccini, E., and Raiteri, M. (2006). Trafficking of presynaptic AMPA receptors mediating neurotransmitter release: neuronal selectivity and relationships with sensitivity to cyclothiazide. $\mathrm{Neu}$ ropharmacology 50, 286-296. doi: 10.1016/j.neuropharm.2005.09.004

Pittaluga, A., Longordo, F., Chiaramonte, G., and Raiteri, M. (2004). HIV-1 Tat protein potentiates presynaptic NMDA receptors releasing noradrenaline from human and rat isolated nerve endings. J. Neurovirol. Taylor Francis 10, 125-126.

Pittaluga, A., Pattarini, R., Feligioni, M., and Raiteri, M. (2001). NMDA receptors mediating hippocampal noradrenaline and striatal dopamine release display differential sensitivity to quinolinic acid, the HIV-1 envelope protein gp120, external pH and PKC inhibition. J. Neurochem. 76, 139-148. doi: 10.1046/j.14714159.2001.00057.x

Pittaluga, A., Raiteri, L., Longordo, F., Luccini, E., Barbiero, V. S., Racagni, G., et al. (2007). Antidepressant treatments and function of glutamate ionotropic receptors mediating amine release in hippocampus. Neuropharmacology 53, 27-36. doi: 10.1016/j.neuropharm. 2007.04.006

Quik, M., O'Neill, M., and Perez, X. A. (2007). Nicotine neuroprotection against nigrostriatal damage: importance of the animal model. Trends Pharmacol. Sci. 28, 229-235. doi: 10.1016/j.tips.2007.03.001

Raiteri, M., Angelini, F., and Levi, G. (1974). A simple apparatus for studying the release of neurotransmitters from synaptosomes. Eur. J. Pharmacol. 25, 411-414. doi: 10.1016/0014-2999(74)90272-6

Raiteri, M., Marchi, M., Maura, G., Bonanno, G., Pittaluga, A., Paudice, P., et al. (1992). "Presynaptic receptors modulating transmitter release: physiological and pharmacological aspects," in Trends in Receptor Research: Proceedings of the 8th Camerino-Noordwijkerhout Symposium, Camerino, Italy, 8-12 September 1991, Vol. 18, eds P. Angeli, U. Gulini, and W. Quaglia (Amsterdam: Elsevier Science Ltd.), 73.

Risso, F., Grilli, M., Parodi, M., Bado, M., Raiteri, M., and Marchi, M. (2004). Nicotine exerts a permissive role on NMDA receptor function in hippocampal noradrenergic terminals. Neuropharmacology 47, 65-71. doi: 10.1016/ j.neuropharm.2004.02.018

Russo, C., Marchi, M., Andrioli, G. C., Cavazzani, P., and Raiteri, M. (1993). Enhancement of glycine release from human brain cortex synaptosomes by acetylcholine acting at M4 muscarinic receptors. J. Pharmacol. Exp. Ther. 266, 142-146. 
Salamone, A., Zappettini, S., Grilli, M., Olivero, G., Agostinho, P., Tomé, A. R., et al. (2014). Prolonged nicotine exposure down-regulates presynaptic NMDA receptors in dopaminergic terminals of the rat nucleus accumbens. Neuropharmacology 79, 488-497. doi: 10.1016/j.neuropharm.2013.12.014

Salminen, O., Drapeau, J. A., McIntosh, J. M., Collins, A. C., Marks, M. J., and Grady, S. R. (2007). Pharmacology of $\alpha$-conotoxin MII-sensitive subtypes of nicotinic acetylcholine receptors isolated by breeding of null mutant mice. Mol. Pharmacol. 71, 1563-1571. doi: 10.1124/mol.106.031492

Sanz-Clemente, A., Nicoll, R. A., and Roche, K. W. (2013). Diversity in NMDA receptor composition: many regulators, many consequences. Neuroscientist 19 , 62-75. doi: 10.1177/1073858411435129

Sershen, H., Balla, A., Lajtha, A., and Vizi, E. S. (1997). Characterization of nicotinic receptors involved in the release of noradrenaline from the hippocampus. Neuroscience 77, 121-130. doi: 10.1016/S0306-4522(96)00425-3

Sher, E., Chen, Y., Sharples, T. J. W., Broad, L. M., Benedetti, G., Zwart, R., et al. (2004). Physiological roles of neuronal nicotinic receptors subtypes: new insights on the nicotinic modulation of neurotransmitter release, synaptic transmission and plasticity. Curr. Top. Med. Chem. 4, 283-297. doi: 10.2174/ 1568026043451393

Shimohama, S. (2009). Nicotinic receptor-mediated neuroprotection in neurodegenerative disease models. Biol. Pharm. Bull. 32, 332-336. doi: 10.1248/bpb. 32.332

Summa, M., Di Prisco, S., Grilli, M., Marchi, M., and Pittaluga, A. (2011). Hippocampal AMPA autoreceptors positively coupled to NMDA autoreceptors traffic in a constitutive manner and undergo adaptative changes following enriched environment training. Neuropharmacology 61, 1282-1290. doi: 10.1016/ j.neuropharm.2011.07.032

Taupin, P., Zini, S., Cesselin, F., Ben-Ari, Y., and Roisin, M. P. (1994). Subcellular fractionation on Percoll gradient of mossy fiber synaptosomes: morphological and biochemical characterization in control and degranulated rat hippocampus. J. Neurochem. 62, 1586-1595. doi: 10.1046/j.1471-4159.1994.62041586.x

Timofeeva, O. A., and Levin, E. D. (2011). Glutamate and nicotinic receptor interactions in working memory: importance for the cognitive impairment of schizophrenia. Neuroscience 195, 21-36. doi: 10.1016/j.neuroscience. 2011.08.038

Tovar, K. R., and Westbrook, G. L. (2002). Mobile NMDA receptors at hippocampal synapses. Neuron 34, 255-264. doi: 10.1016/S0896-6273(02)00658-X

Trauth, J. A., Seidler, F. J., and Slotkin, T. A. (2000). An animal model of adolescent nicotine exposure: effects on gene expression and macromolecular constituents in rat brain regions. Brain Res. 867, 29-39. doi: 10.1016/S0006-8993(00)02208-3

Turner, T. J. (2004). Nicotine enhancement of dopamine release by a calciumdependent increase in the size of the readily releasable pool of synaptic vesicles. J. Neurosci. 24, 11328-11336. doi: 10.1523/JNEUROSCI.1559-04.2004
Vibat, C. R., Lasalde, J. A., McNamee, M. G., and Ochoa, E. L. (1995). Differential desensitization properties of rat neuronal nicotinic acetylcholine receptor subunit combinations expressed in Xenopus laevis oocytes. Cell. Mol. Neurobiol. 15, 411-425. doi: 10.1007/BF02071877

Vizi, E. S., and Lendvai, B. (1999). Modulatory role of presynaptic nicotinic receptors in synaptic and non-synaptic chemical communication in the central nervous system. Brain Res. Brain Res. Rev. 30, 219-235. doi: 10.1016/S01650173(99)00016-8

Wonnacott, S. (1997). Presynaptic nicotinic ACh receptors. Trends Neurosci. 20, 92-98. doi: 10.1016/S0166-2236(96)10073-4

Zappettini, S., Grilli, M., Lagomarsino, F., Cavallero, A., Fedele, E., and Marchi, M. (2011). Presynaptic nicotinic $\alpha 7$ and non- $\alpha 7$ receptors stimulate endogenous GABA release from rat hippocampal synaptosomes through two mechanisms of action. PLoS ONE 6:e16911. doi: 10.1371/journal.pone.0016911

Zappettini, S., Grilli, M., Olivero, G., Chen, J., Padolecchia, C., Pittaluga, A., et al. (2014). Nicotinic $\alpha 7$ receptor activation selectively potentiates the function of NMDA receptors in glutamatergic terminals of the nucleus accumbens. Front. Cell. Neurosci. 8:332. doi: 10.3389/fncel.2014.00332

Zappettini, S., Grilli, M., Salamone, A., Fedele, E., and Marchi, M. (2010). Presynaptic nicotinic receptors evoke endogenous glutamate and aspartate release from hippocampal synaptosomes by way of distinct coupling mechanisms. $\mathrm{Br}$. J. Pharmacol. 161, 1161-1171. doi: 10.1111/j.1476-5381.2010.00958.x

Zhong, C., López-Hernández, G. Y., Talmage, D. A., and Role, L. W. (2014). "Presynaptic nicotinic acetylcholine receptors and the modulation of circuit excitability," in Nicotinic Receptors, ed. R. A. J. Lester (New York: Springer), 137-167. doi: 10.1007/978-1-4939-1167-7_7

Zhou, X., Chen, Z., Yun, W., and Wang, H. (2015). NMDA receptor activity determines neuronal fate: location or number? Rev. Neurosci. 26, 39-47. doi: 10.1515/revneuro-2014-0053

Zoli, M., Moretti, M., Zanardi, A., McIntosh, J. M., Clementi, F., and Gotti, C. (2002). Identification of the nicotinic receptor subtypes expressed on dopaminergic terminals in the rat striatum. J. Neurosci. 22, 8785-8789.

Conflict of Interest Statement: The authors declare that the research was conducted in the absence of any commercial or financial relationships that could be construed as a potential conflict of interest.

Copyright (C) 2015 Marchi, Grilli and Pittaluga. This is an open-access article distributed under the terms of the Creative Commons Attribution License (CC BY). The use, distribution or reproduction in other forums is permitted, provided the original author(s) or licensor are credited and that the original publication in this journal is cited, in accordance with accepted academic practice. No use, distribution or reproduction is permitted which does not comply with these terms. 